\title{
Research on the Constitutive Behavior of the Sintered Reactive Material PTFE/Al/Si
}

\author{
Xianwen Ran, Wenhui Tang \\ College of Liberal Arts and Sciences, National University of Defense Technology, Changsha, China \\ Email: ranxianwen@163.com
}

How to cite this paper: Ran, X.W. and Tang, W.H. (2021) Research on the Constitutive Behavior of the Sintered Reactive Material PTFE/Al/Si. Journal of Materials Science and Chemical Engineering, 9, 70-82. https://doi.org/10.4236/msce.2021.910005

Received: September 27, 2021

Accepted: October 26, 2021

Published: October 29, 2021

\begin{abstract}
The constitutive behaviors of the sintered reactive material PTFE/Al/Si, one new formulation proposed by the author, were studied systematically and found it appears strain-harden, stain-rate harden and temperature-soften effects, so it is more appropriate to describe the mechanical behavior with the Johnson-Cook model. With the static and dynamic experimental results and using the nonlinear fitting method, the mechanical and physical parameters in the Johnson-Cook model were determined, which supply one basis for the future numerical simulation study.
\end{abstract}

\section{Keywords}

Reactive Material, PTFE/Al/Si, Johnson-Cook Model, Static Experiment, Dynamic Experiment

\section{Introduction}

Reactive material is a new energetic material which is widely studied and used at present, and first discovered in 1970s [1]. In recent years, the metal/polymer mixture type reactive materials have been fully valued by various military powers, especially the reactive material slotted on fluoropolymer and reactive materials, which has shown great superiority in the application of weapons and equipment [2]. Taking PTFE/Al (73.5\%/26.5\%) reactive material as an example, it is unit mass energy and unit volume energy can reach 3.5 times and 5 times TNT explosives, and the chemical energy released by the reaction under impact load can reach dozens of times its own kinetic energy [3]. Under the condition of high-speed impact loading, PTFE/Al will undergo violent combustion and detonation reaction, and the reaction rate is roughly between the combustion rate of propellant and the detonation rate of explosive [4] [5]. The heat released by the unit mass reaction of PTFE/Al (wt\%: 73.5/26.5) is about $8.53 \mathrm{~kJ} / \mathrm{g}$, which 
is about 2 times of TNT explosive under the same conditions. The energy release rate of PTFE/Al reactive material during reaction mainly depends on the external impact loading speed, the higher the impact loading speed is, the higher the energy release rate of the material is [6].

Compared with the conventional explosive, the sintered PTFE/Al reactive material can have a high tensile-strength up to about $20 \mathrm{MPa}$, but which is merely about one tenth of the tensile strength that the usual structural materials (such as aluminum) have. To assess the structural ability of PTFE/Al, many works have been done. Cai [7] [8] carried out a large number of mechanical properties tests on PTFE/Al, including quasi-static compression test, drop impact test, dynamic SHPB test. The results show that when the particle size of Al powder is 2 $\mu \mathrm{m}$, a small part of PTFE/Al sample will react in the process of quasi-static compression. Osborne [9] and Mock [1] [10] carried out drop impact test and Taylor impact test for $\mathrm{PTFE} / \mathrm{Al}$ reactive materials, and the influence of $\mathrm{Al}$ particle size on the energy release characteristics of PTFE/Al reactive materials was studied. It is found that the PTFE/Al reactive materials with smaller $\mathrm{Al}$ particles are more likely to react, because the smaller Al particle size means that the specific surface area of the particles is larger, and the initial energy required to induce the reaction is less.

Recently, based on the PTFE/Al, one new formulation PTFE/Al/Si was presented and the energy-released experiments showed it had a less stimulated energy and a better ability to do external work than that PTFE/Al had, which indicated that PTFE/Al/Si is more appropriate to be used as reactive fragments [11]. To assess its structural ability further, the static and dynamic experiments had been done systematically in this paper, and from which the mechanical and physical parameters in Johnson-Cook model (JC model) [12] were derived, which will be greatly helpful for numerical simulation and can present the behavior depended on strain-rate.

\section{Experimental Results}

\subsection{Static Results}

The samples used in the static experiments are shown in Figure 1 and their dimensions are given in Table 1. During the experiments, the loading strain rate is $0.001 \mathrm{~s}^{-1}$.

The engineering stress-engineering strain curves measured in the static experiments were shown in Figure 2.

From Figure 2, the real stress and the real strain for the sample in the static experiment can be derived with the Equation (1).

$$
\sigma=\left(1-\varepsilon_{\text {eng }}\right) \sigma_{\text {eng }} ; \quad \varepsilon=-\ln \left(1-\varepsilon_{\text {eng }}\right)
$$

The derived the real stress-the real strain curves were shown in Figure 3.

It can be seen from Figure 3, under compression load, the sintered PTFE/Al/Si show one elastic segment and one plastic segment. In the plastic segment, there 
is an obvious strain hardening behavior. From Figure 3, the elastic-modulus, strain harden modulus, nominal yield strength and the yield strain can be calculated and shown in Table 2.

Table 1. The dimensional parameters of the samples used in static experiments.

\begin{tabular}{cccc}
\hline Num. & Mass $/(\mathrm{g})$ & Diameter $/(\mathrm{mm})$ & Height $/(\mathrm{mm})$ \\
\hline$\# 5-1$ & 1.736 & 9.92 & 10.02 \\
$\# 5-2$ & 1.735 & 9.92 & 9.98 \\
$\# 5-3$ & 1.736 & 9.92 & 10.01 \\
\hline
\end{tabular}

Table 2. The dimensional parameters of the samples used in static experiments.

\begin{tabular}{ccccc}
\hline Num. & $\begin{array}{c}\text { Elastic-Modulus } \\
\text { E/(MPa) }\end{array}$ & $\begin{array}{c}\text { Strain-harden } \\
\text { Modulus Es/(MPa) }\end{array}$ & $\begin{array}{c}\text { Nominal Yield } \\
\text { Strength } \sigma_{\mathrm{s}} /(\mathrm{MPa})\end{array}$ & $\begin{array}{c}\text { Yield } \\
\text { Strain } \varepsilon_{\mathrm{s}}\end{array}$ \\
\hline$\# 5-1$ & 387.2 & 2.7 & 12.9 & 0.0546 \\
$\# 5-2$ & 375.9 & 3.8 & 12.9 & 0.0556 \\
$\# 5-3$ & 369.6 & 3.0 & 13.0 & 0.0526 \\
\hline
\end{tabular}

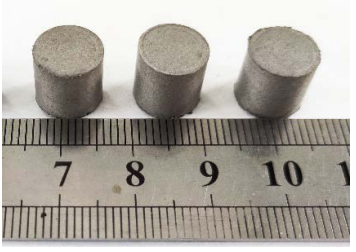

(a)

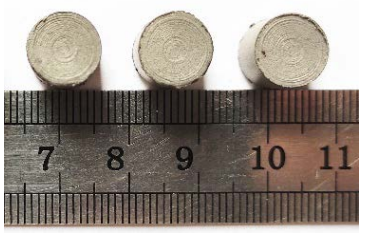

(b)

Figure 1. The samples used in the static experiments. (a) Front view; (b) Top view.
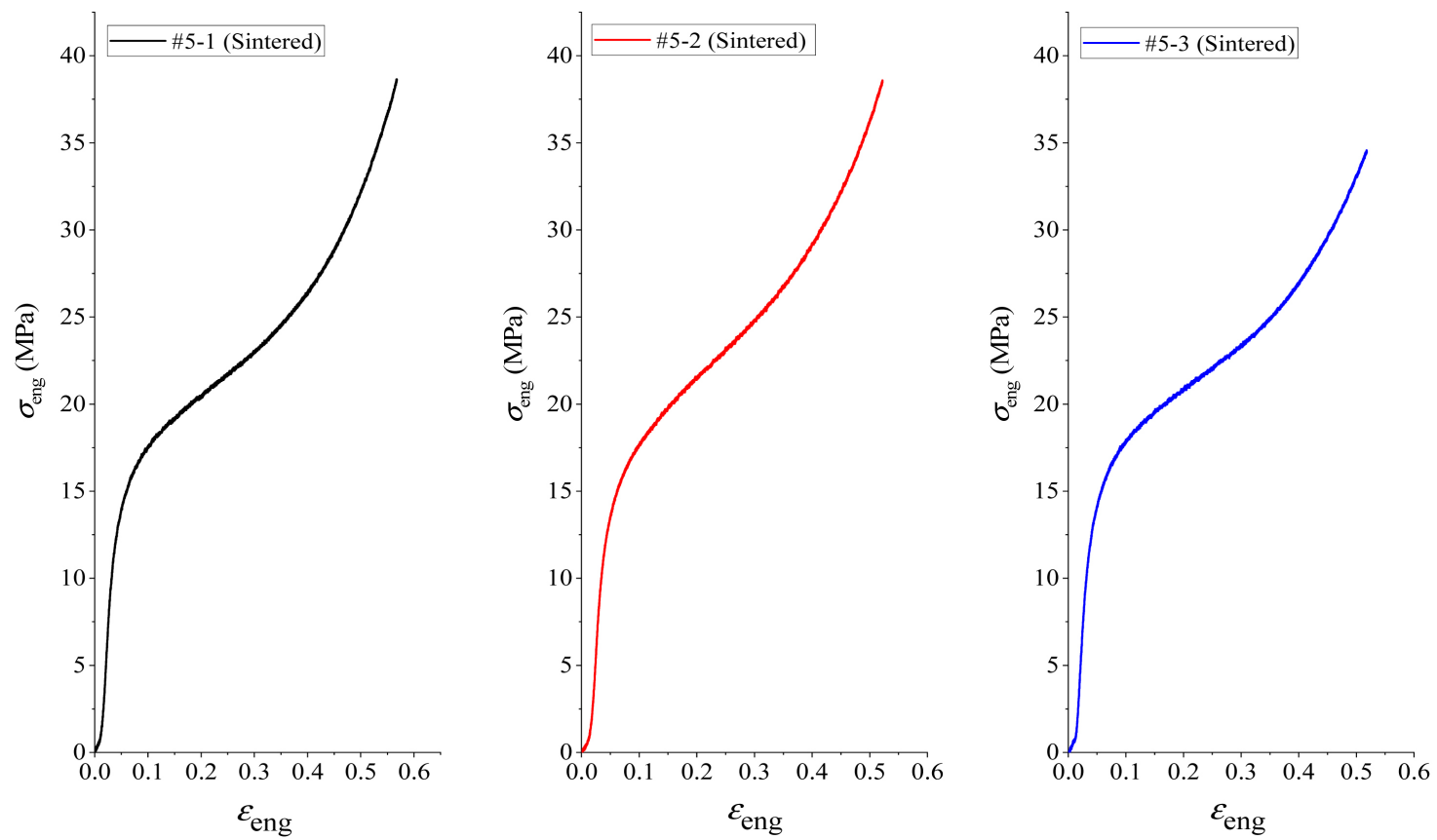

Figure 2. The engineering stress-engineering strain curves for the sintered PTFE/Al/Si. 


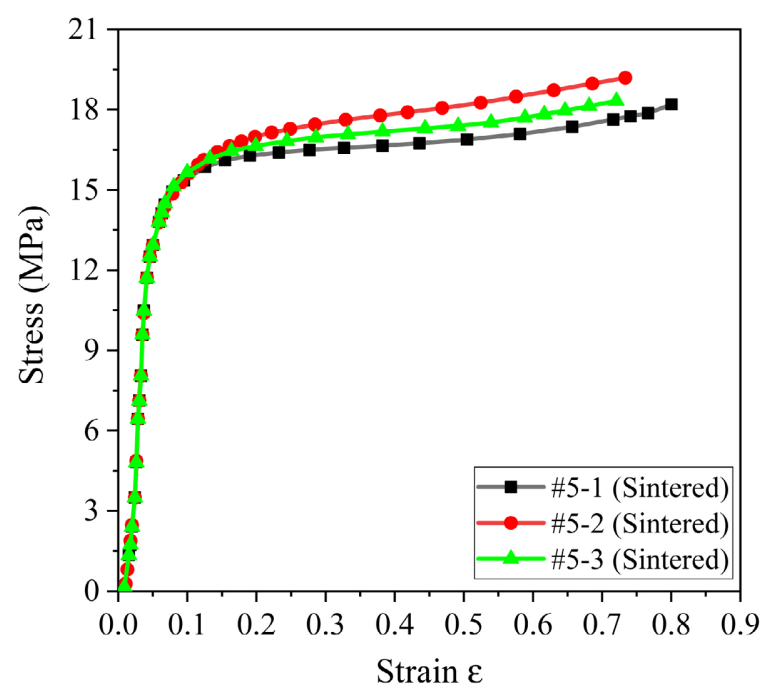

Figure 3. The real stress-real strain curves of the sintered PTFE/Al/Si.

\subsection{Dynamic Results}

The dynamical behavior of PTFE/Al/Si can be studied with the split Hopkinson pressure bars (SHPB), on which the mechanical behaviors under different strain-rates and different temperatures can be tested.

\subsubsection{The Effect of Different Strain Rate}

On SHPB, three strain-rates $3500 \mathrm{~s}^{-1}, 4500 \mathrm{~s}^{-1}$, and $5500 \mathrm{~s}^{-1}$ are chosen after many trials to test the dynamical behavior of the sintered samples, and to enhance the validity of the experimental results, each strain-rate is repeated in the experiments for three times. The environmental temperature is about $25^{\circ} \mathrm{C}$, and the dimensional parameters of the samples used in experiments are shown in Table 3.

The real stress-real-strain curves of the sintered PTFE/Al/Si under different strain rates were shown in Figure 4.

From the real stress-real strain curves in Figure 4, the main dynamic mechanical parameters, such as strain-harden modulus, yield strength and critical strain (which indicates the strain corresponding with the summit stress), are derived and shown in Table 4.

Compared the values of the strain-harden modulus and yield strength in both Table 3 and Table 4, we can see that the mechanical behaviors of the sintered reactive material $\mathrm{PTFE} / \mathrm{Al} / \mathrm{Si}$ are sensitive to the strain-rate and the sintered reactive material $\mathrm{PTFE} / \mathrm{Al} / \mathrm{Si}$ is a strain-rate harden material, which indicates it is appropriate to describe its mechanical behavior by using the JC model.

\subsubsection{The Effect of Different Temperature}

The main component of PTFE/Al/Si is PTFE which is a type of plastics and has an obvious temperature-soften behavior. So, it is needed to study the dynamical behavior of $\mathrm{PTFE} / \mathrm{Al} / \mathrm{Si}$ under different temperature. In the experiments, the strain-rate is about $4500 \mathrm{~s}^{-1}$, and the four temperatures chosen are $25^{\circ} \mathrm{C}, 100^{\circ} \mathrm{C}$, 


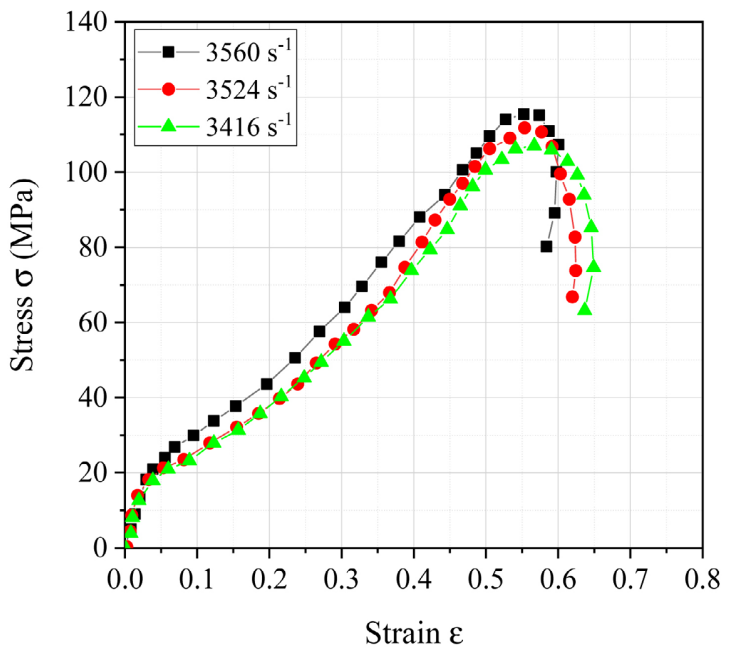

(a)

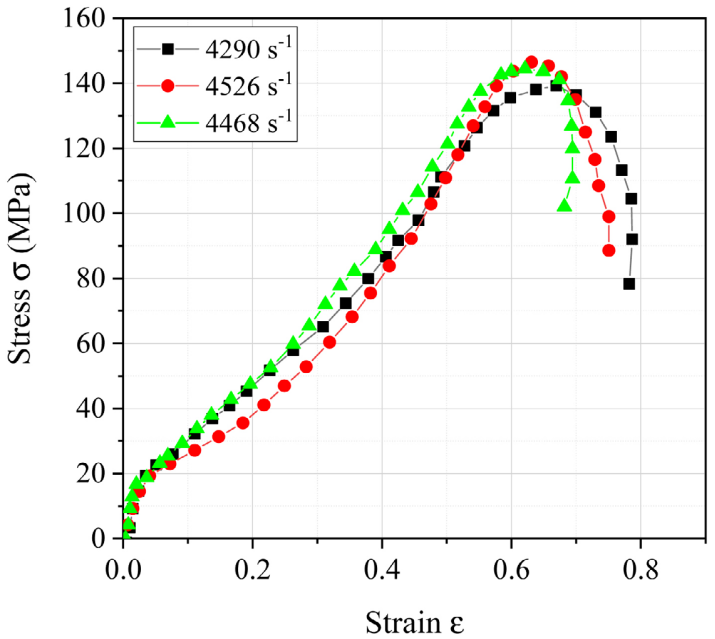

(b)

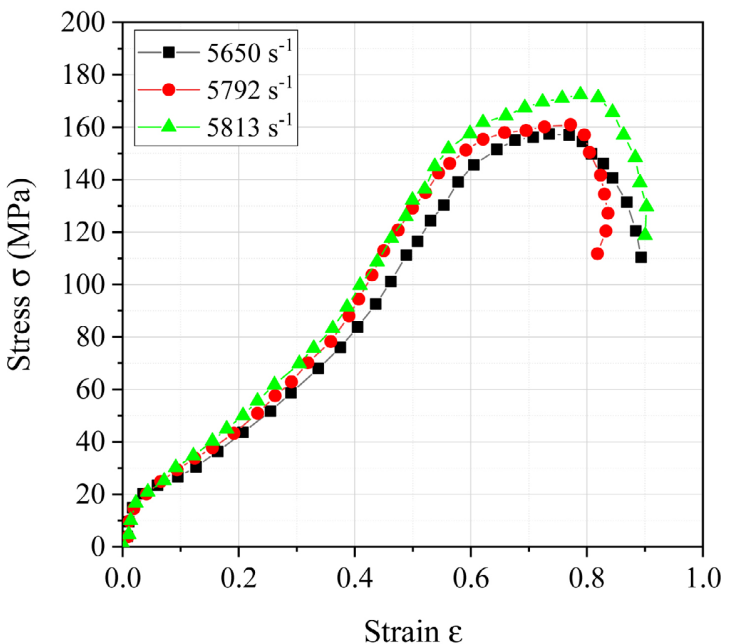

(c)

Figure 4. The real stress-real strain curves of the sintered PTFE/Al/Si under different strain-rates. (a) Strain-rate $3500 \mathrm{~s}^{-1}$; (b) Strain-rate $4500 \mathrm{~s}^{-1}$; (c) Strain-rate $5500 \mathrm{~s}^{-1}$. 
Table 3. The dimensional parameters of the samples used in dynamic experiments.

\begin{tabular}{ccccc}
\hline Num. & Mass $/(\mathrm{g})$ & Diameter $/(\mathrm{mm})$ & Height $/(\mathrm{mm})$ & Strain-rate $/\left(\mathrm{s}^{-1}\right)$ \\
\hline$\# 1$ & 0.186 & 6.04 & 3.10 & 3500 \\
$\# 2$ & 0.181 & 6.04 & 3.08 & 3500 \\
$\# 3$ & 0.188 & 6.04 & 3.10 & 3500 \\
$\# 4$ & 0.204 & 6.04 & 3.11 & 4500 \\
$\# 5$ & 0.196 & 6.04 & 3.05 & 4500 \\
$\# 6$ & 0.197 & 6.04 & 3.06 & 4500 \\
$\# 7$ & 0.196 & 6.04 & 3.02 & 5500 \\
$\# 8$ & 0.189 & 6.04 & 3.06 & 5500 \\
$\# 9$ & 0.195 & 6.04 & 2.92 & 5500 \\
\hline
\end{tabular}

Table 4. The mechanical parameters derived from the dynamic experiments.

\begin{tabular}{cccc}
\hline $\begin{array}{c}\text { Strain-rate } \\
\dot{\varepsilon} /\left(\mathrm{s}^{-1}\right)\end{array}$ & $\begin{array}{c}\text { Strain-harden Modulus } \\
\text { Ed/(MPa) }\end{array}$ & $\begin{array}{c}\text { Yield Strength } \\
\sigma \mathrm{d} /(\mathrm{MPa})\end{array}$ & $\begin{array}{c}\text { Critical } \\
\text { Strain } \varepsilon \mathrm{c}\end{array}$ \\
\hline 3416 & 174 & 17.9 & 0.5523 \\
3524 & 180 & 18.4 & 0.5533 \\
3560 & 185 & 18.2 & 0.5706 \\
4290 & 204 & 18.5 & 0.6593 \\
4468 & 206 & 18.8 & 0.6210 \\
4526 & 209 & 19.2 & 0.6312 \\
5650 & 211 & 19.1 & 0.7348 \\
5792 & 219 & 19.5 & 0.7519 \\
5813 & 227 & 20.1 & 0.7880 \\
\hline
\end{tabular}

$150^{\circ} \mathrm{C}, 200^{\circ} \mathrm{C}$. The dimensional parameters of the samples in this set of experiments are shown in Table 5 .

The dynamical real stress-real strain curves for the sintered PTFE/Al/Si under four temperatures were shown in Figure 5.

It can be seen from the comparison Figures 5(a)-(d), that the ultimate compressive strength of the sintered reactive material PTFE/Al/Si decreases with the temperature increasing, which indicates the mechanical behavior of PTFE/Al/Si has a temperature-soften characteristic. This specialty can also be described by the JC model.

\section{Determination of the Parameters in the JC Model}

JC model was proposed in 1983 and has been widely used in the field of the impact or explosion dynamics. The form embedded in the Ls-dyna was

$$
\sigma_{y}=\left(A+B \bar{\varepsilon}_{p}^{n}\right)\left[1+C \ln \left(\dot{\varepsilon} / \dot{\varepsilon}_{0}\right)\right]\left[1-\left(\frac{T-T_{r}}{T_{m}-T_{r}}\right)^{m}\right]
$$


Table 5. The dimensional parameters of the samples used in dynamic experiments.

\begin{tabular}{ccccc}
\hline Num. & Mass/(g) & Diameter $/(\mathrm{mm})$ & Height $/(\mathrm{mm})$ & Temperature $/\left({ }^{\circ} \mathrm{C}\right)$ \\
\hline$\# 4$ & 0.204 & 6.04 & 3.11 & 25 \\
$\# 5$ & 0.196 & 6.04 & 3.05 & 25 \\
$\# 6$ & 0.197 & 6.04 & 3.06 & 25 \\
$\# 10$ & 0.191 & 6.04 & 3.08 & 100 \\
$\# 11$ & 0.190 & 6.04 & 3.11 & 100 \\
$\# 12$ & 0.189 & 6.04 & 3.10 & 100 \\
$\# 13$ & 0.189 & 6.04 & 3.07 & 150 \\
$\# 14$ & 0.195 & 6.04 & 3.08 & 150 \\
$\# 15$ & 0.187 & 6.04 & 3.05 & 150 \\
$\# 16$ & 0.184 & 6.04 & 3.03 & 200 \\
$\# 17$ & 0.187 & 6.04 & 3.08 & 200 \\
$\# 18$ & 0.185 & 6.04 & 3.04 & 200 \\
\hline
\end{tabular}

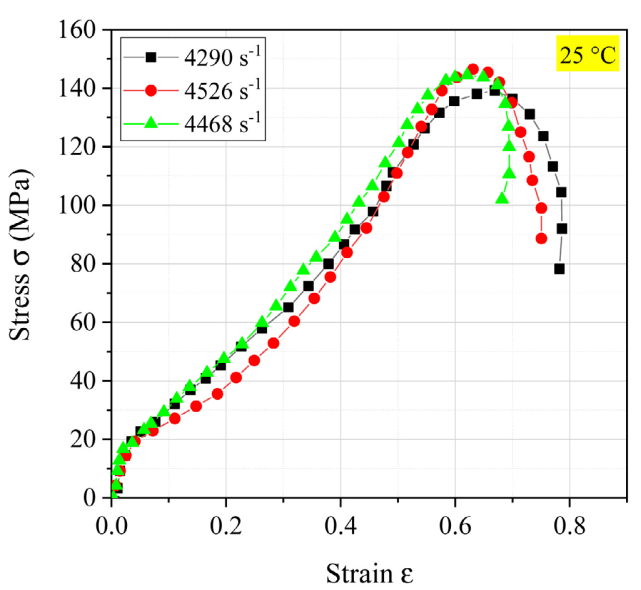

(a)

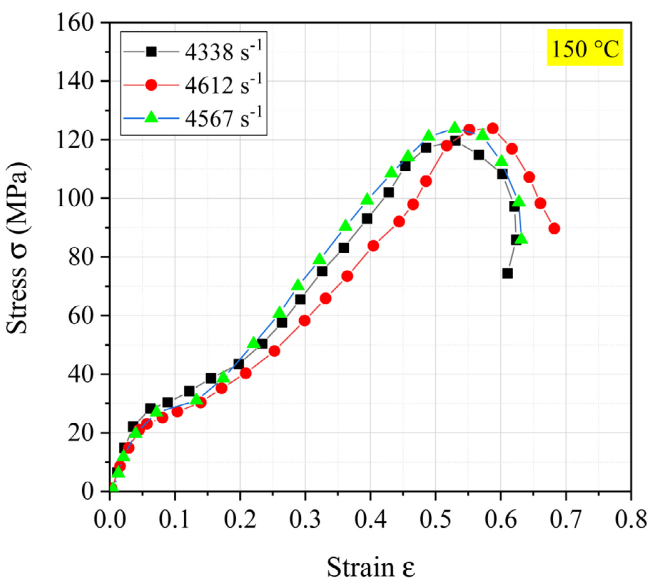

(c)

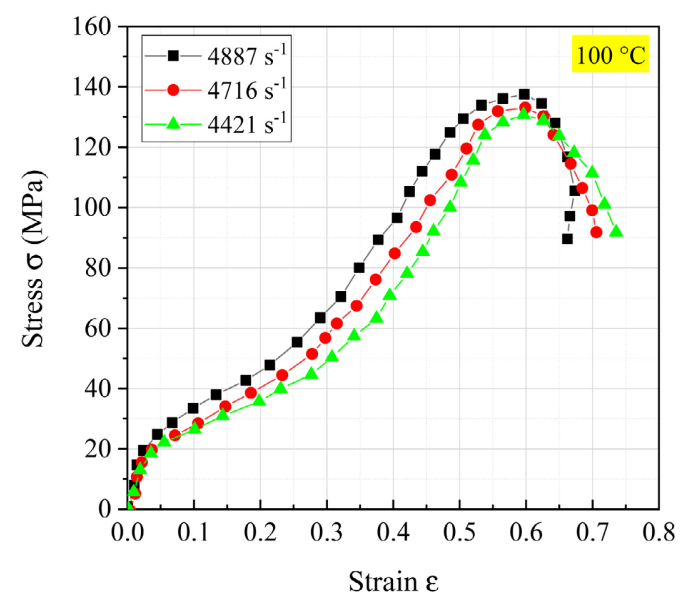

(b)

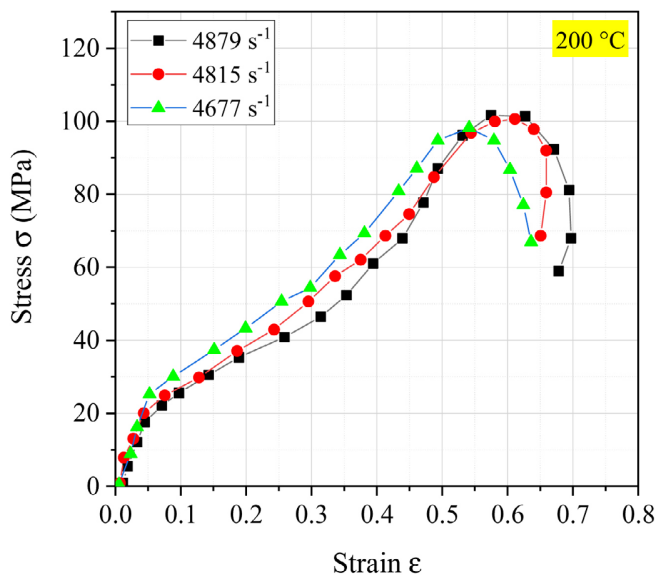

(d)

Figure 5. The real stress-real strain curves of the sintered PTFE/Al/Si under different temperatures. (a) $25^{\circ} \mathrm{C}$; (b) $100^{\circ} \mathrm{C}$; (c) $150^{\circ} \mathrm{C}$; (d) $200^{\circ} \mathrm{C}$. 
where $A$ is the yield strength, $B$ the strain-harden coefficient, $n$ the strain-harden index, $C$ the strain-rate harden coefficient, $m$ the temperature-soften index, $\bar{\varepsilon}_{p}$ the effective plastic strain, $\dot{\varepsilon}_{0}$ the reference strain-rate, $T_{r}$ the environmental temperature, $T_{m}$ the melting temperature.

It is obvious that, in JC model, there are totally three terms corresponding respectively to the strain-harden, the strain-rate harden and the temperature-soften terms, which needs to be considered in the derivation of the parameters above.

\subsection{The Strain-Harden Term}

If choosing $25^{\circ} \mathrm{C}$ as the environmental temperature and the static strain-rate $0.001 \mathrm{~s}^{-1}$ as the reference strain-rate, when using the static experimental results (that is Figure 3), both the strain-rate harden and the temperature-soften terms will equal 1 and then the JC model in the Equation (2) will be simplified to the following form

$$
\sigma_{y}=\left(A+B \bar{\varepsilon}_{p}^{n}\right)
$$

From the real stress-real strain curves in the Figure 3, we can pick the plastic stage up and plot them in Figure 6(a), then we can do no linear fitting for them with the Equation (3). The fitting curves are shown in Figures 6(b)-(d), and the determined parameters in the Equation (3) are shown in Table 6.

From Figure 6, it can be seen that all the fitting curves fit the experimental data well, and all the coefficients of determination (COD) $\mathrm{R}^{2}$ are close to 1 . Of all the three experimental curves, the \#5-3 approaches the average of three most closely, so we can pick the fitting results of \#5-3 as the mechanical parameters in the plastic stage for the sintered PTFE/Al/Si, that is

$$
\sigma_{y}=13.0+5.949 \bar{\varepsilon}_{p}^{0.289}
$$

\subsection{The Strain-Rate Harden Term}

According to the Equation (2), if choose the $25^{\circ} \mathrm{C}$ as the environmental temperature, the Equation (2) will change to

$$
\sigma_{y}=\left(A+B \bar{\varepsilon}_{p}^{n}\right)\left[1+C \ln \left(\dot{\varepsilon} / \dot{\varepsilon}_{0}\right)\right]
$$

In the Equation (5), the dependence of stress on the strain-rate is expressed as a logarithmic form, which is enough to describe the metallic material but is insufficient for the polymer such as PTFE because polymers have a high viscosity which is usually more dependent on the strain-rate. Xu [13] had studied the

Table 6. The dimensional parameters of the samples used in static experiments.

\begin{tabular}{ccccc}
\hline Num. & $A /(\mathrm{MPa})$ & $B /(\mathrm{MPa})$ & $n$ & $R^{2}$ \\
\hline $5-1$ & 12.9 & 5.349 & 0.282 & 0.96777 \\
$\# 5-2$ & 12.9 & 7.138 & 0.333 & 0.97859 \\
$\# 5-3$ & 13.0 & 5.949 & 0.289 & 0.97174 \\
\hline
\end{tabular}




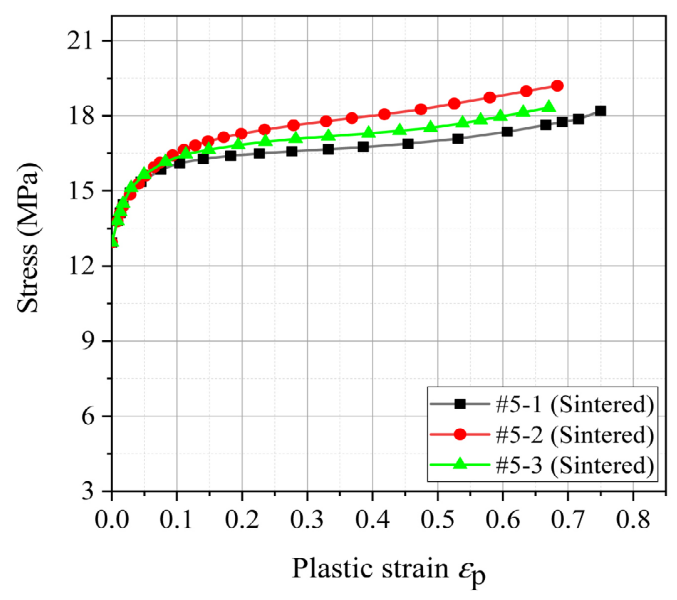

(a)

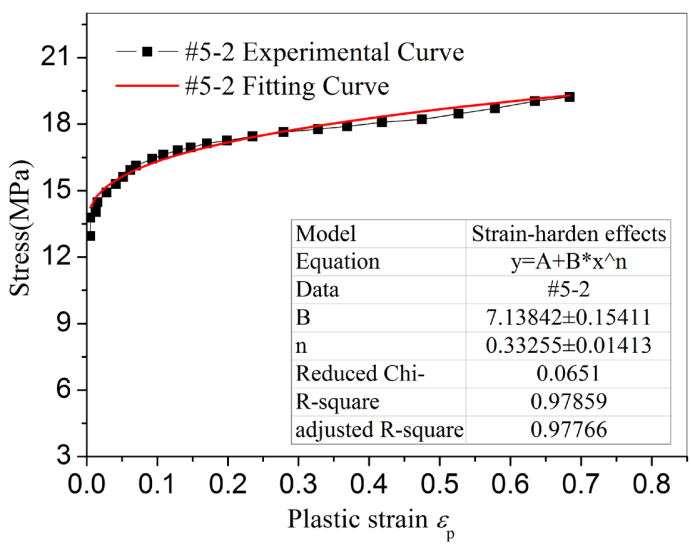

(c)

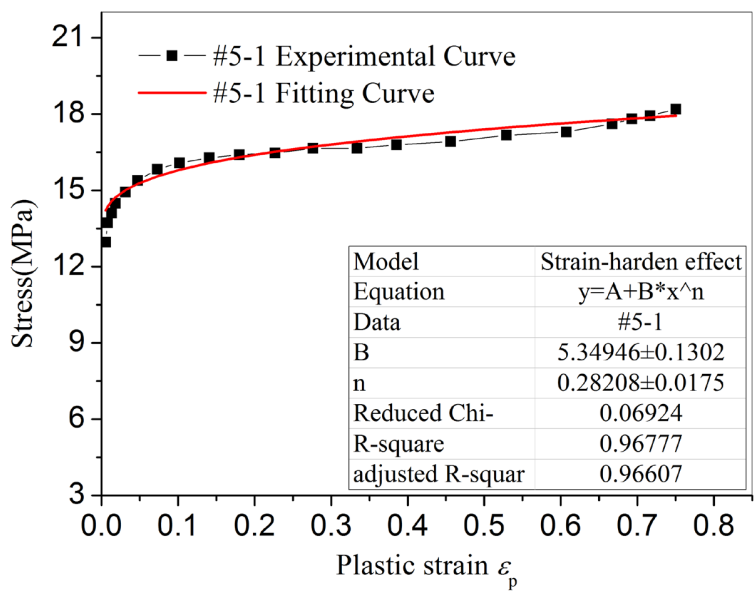

(b)

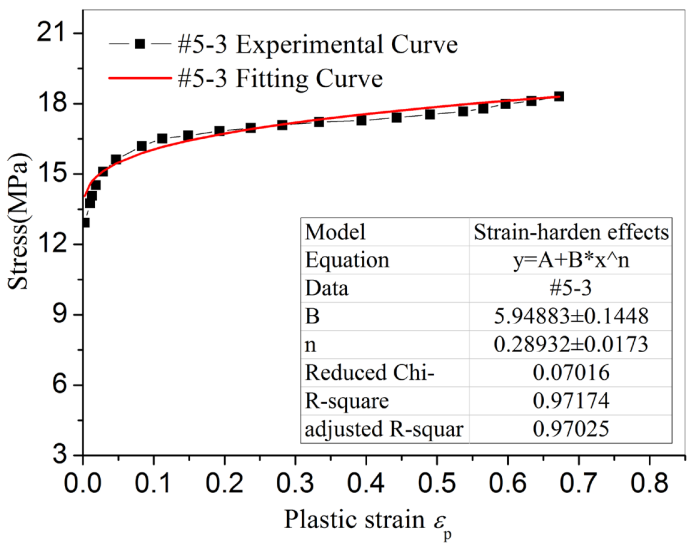

(d)

Figure 6. The fitting curves for the strain-harden term of the sintered PTFE/Al/Si. (a) The experimental real stress-real strain curves in the plastic stage; (b) The fitting curve in the plastic stage for \#5-1; (c) The fitting curve in the plastic stage for \#5-2; (d) The fitting curve in the plastic stage for \#5-3.

strain-rate harden behaviors on the sintered PTFE/Al and found that, with the increase of the strain-rate, the plastic stress will increase quickly, but the dependence of plastic stress on the strain-rate will become weaker and weaker, which indicates it is more appropriate to describe the dependence of the plastic stress on the strain-rate by choosing the exponential form as the following.

$$
\sigma_{y}=\left(A+B \bar{\varepsilon}_{p}^{n}\right)\left(\dot{\varepsilon} / \dot{\varepsilon}_{0}\right)^{\lambda}
$$

Based on Figure 4 and taking values of the plastic stresses corresponding respectively to the plastic strains 0.25 and 0.35 , the dependence of the plastic stress on the strain rate can be gotten as shown in Table 7.

With the values in Table 7 and taking the reference strain-rate $0.001 \mathrm{~s}^{-1}$, the value of the parameter $\lambda$ can be determined by using the exponential fitting as shown in the Equation (6). The results are shown in Figure 7.

Compare the fitting results at the two fix plastic-strain, the COD are 0.97631 and 0.95283 respectively which means the fitting result at the plastic-strain 0.25 is more reliable than that at the plastic-strain 0.35 . In addition, according to the 


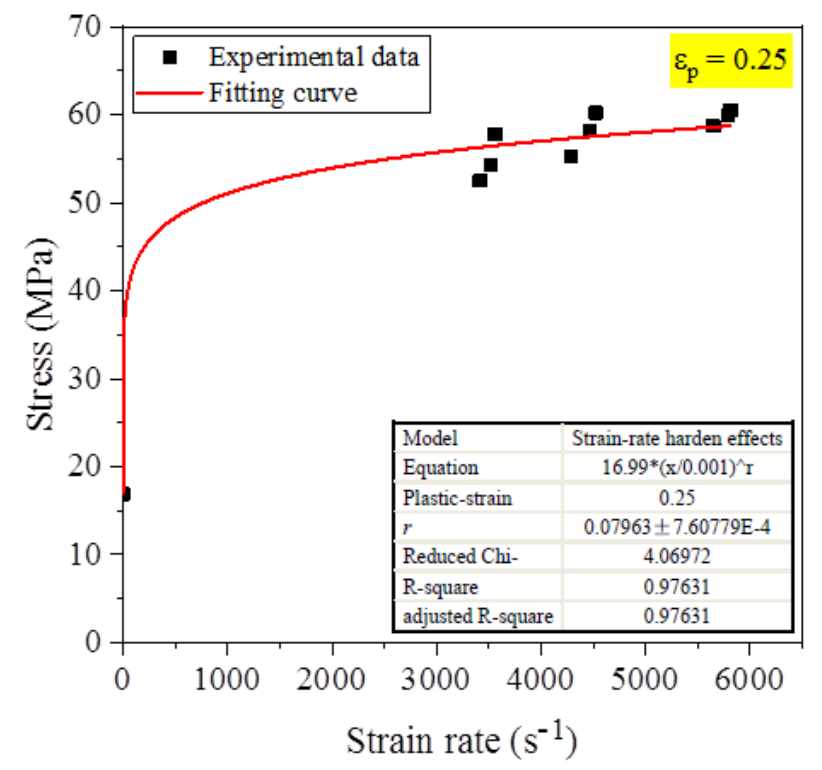

(a)

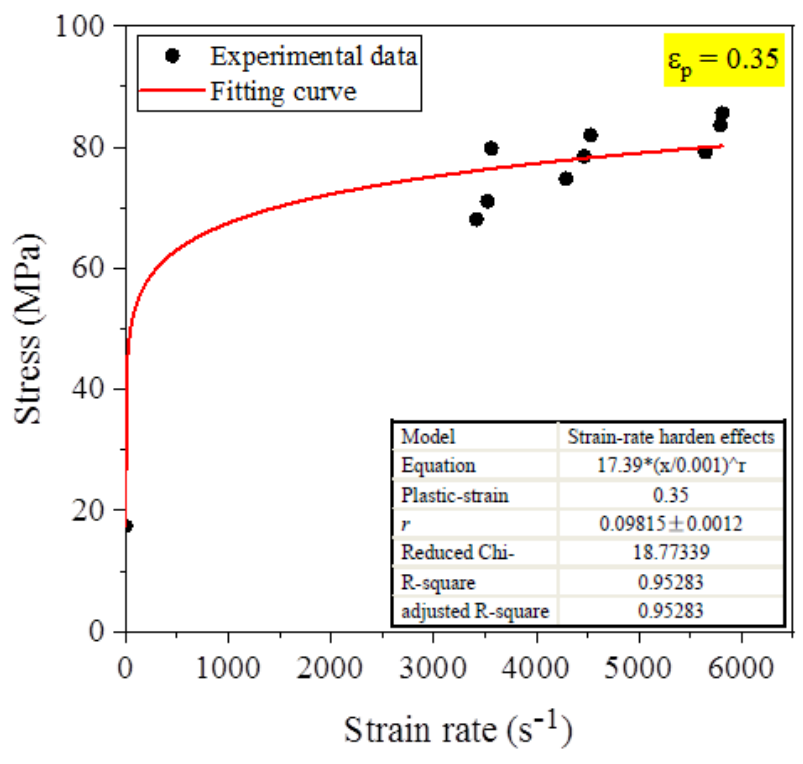

(b)

Figure 7. The fitting curves for the strain-rate harden term at the fix plastic strain. (a) $\varepsilon_{p}=0.25$; (b) $\varepsilon_{p}=0.35$.

Table 7. The dependence of the plastic stress on the strain-rate.

\begin{tabular}{ccc}
$\begin{array}{c}\text { Strain-rate } \\
\dot{\varepsilon} /\left(\mathrm{s}^{-1}\right)\end{array}$ & $\begin{array}{c}\text { The plastic-stress corresponding } \\
\text { to the plastic strain } 0.25 \\
\sigma_{p \mid \varepsilon_{p}=0.25} /(\mathrm{MPa})\end{array}$ & $\begin{array}{c}\text { The plastic-stress corresponding } \\
\text { to the plastic strain } 0.35 \\
\sigma_{p \mid \varepsilon_{p}=0.35} /(\mathrm{MPa})\end{array}$ \\
\hline 0.001 & 16.9 & 17.4 \\
3416 & 52.5 & 68.1 \\
3524 & 54.3 & 71.1 \\
3560 & 57.8 & 79.8 \\
4290 & 55.3 & 74.8 \\
4468 & 58.1 & 78.5 \\
4526 & 60.2 & 82.0 \\
5650 & 58.7 & 79.2 \\
5792 & 59.9 & 83.7 \\
5813 & 60.5 & 85.6 \\
\hline
\end{tabular}

Xu's study [13], the strain-rate harden effect is more obvious at the lower plastic strain. So, it is more appropriate to take the value of the parameter $\lambda$ determined at the plastic-strain 0.25 for the sintered PTFE/Al/Si, that is

$$
\sigma_{y}=\left(13.0+5.949 \bar{\varepsilon}_{p}^{0.289}\right)\left(\dot{\varepsilon} / \dot{\varepsilon}_{0}\right)^{0.07963}
$$

\subsection{The Temperature-Soften Term}

For PTFE is the main component in the sintered reactive material PTFE/Al/Si and the melting temperature of PTFE is about $327^{\circ} \mathrm{C}$, the melting temperatures of $\mathrm{Al}$ and $\mathrm{Si}$ are both above $327^{\circ} \mathrm{C}$, so we can take $327^{\circ} \mathrm{C}$ as the melting temper- 
ature of the sintered PTFE/Al/Si. By taking the values of the plastic stress corresponding to the plastic-strain 0.25 and 0.35 in Figure 5, the dependence of the plastic-stress on the temperature can be gotten and is shown in Table 8.

With the determined parameters $A, B, n$ and $\lambda$, and fitting the temperature-soften term to the values in Table $\mathbf{8}$, the parameter $\mathrm{m}$ can be determined. The results are shown in Figure 8.

Compare the fitting results at the two-fix plastic-strain, the COD are 0.91084 and 0.94312 respectively which means the fitting result at the plastic-strain 0.35 is more reliable than that at the plastic-strain 0.25 . In addition, for those polymers including PTFE, the temperature-soften effect appears more obviously at large plastic strain. So, it is more appropriate to take the value of the parameter $\mathrm{m}$ determined at the plastic-strain 0.35 for the sintered PTFE/Al/Si, that is, the final form of JC model for the sintered PTFE/Al/Si is

$$
\sigma_{y}=\left(13.0+5.949 \bar{\varepsilon}_{p}^{0.289}\right)\left(\dot{\varepsilon} / \dot{\varepsilon}_{0}\right)^{0.07963}\left[1-\left(\frac{T-T_{r}}{T_{m}-T_{r}}\right)^{2.20907}\right]
$$

Table 8. The dependence of the plastic stress on the temperature.

\begin{tabular}{ccc}
\hline $\begin{array}{c}\text { Temperature } \\
T /\left({ }^{\circ} \mathrm{C}\right)\end{array}$ & $\begin{array}{c}\text { The plastic-stress corresponding } \\
\text { to the plastic strain } 0.25 \\
\sigma_{p \mid \varepsilon_{p}=0.25} /(\mathrm{MPa})\end{array}$ & $\begin{array}{c}\text { The plastic-stress corresponding } \\
\text { to the plastic strain } 0.35 \\
\sigma_{p \mid \varepsilon_{p}=0.35} /(\mathrm{MPa})\end{array}$ \\
\hline 25 & 60.2 & 82.0 \\
100 & 56.5 & 78.5 \\
150 & 51.3 & 71.2 \\
200 & 47.3 & 61.7 \\
\hline
\end{tabular}

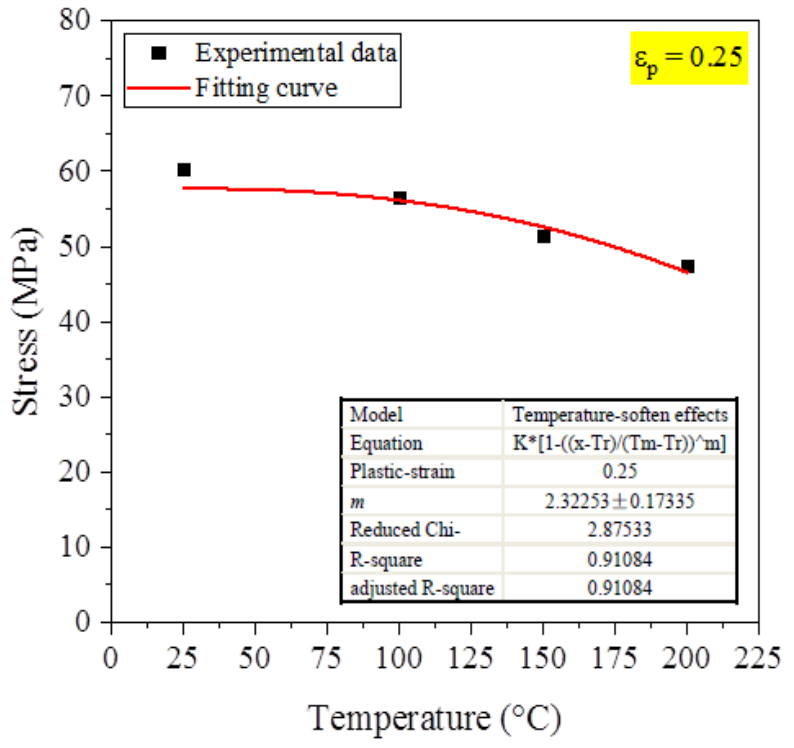

(a)

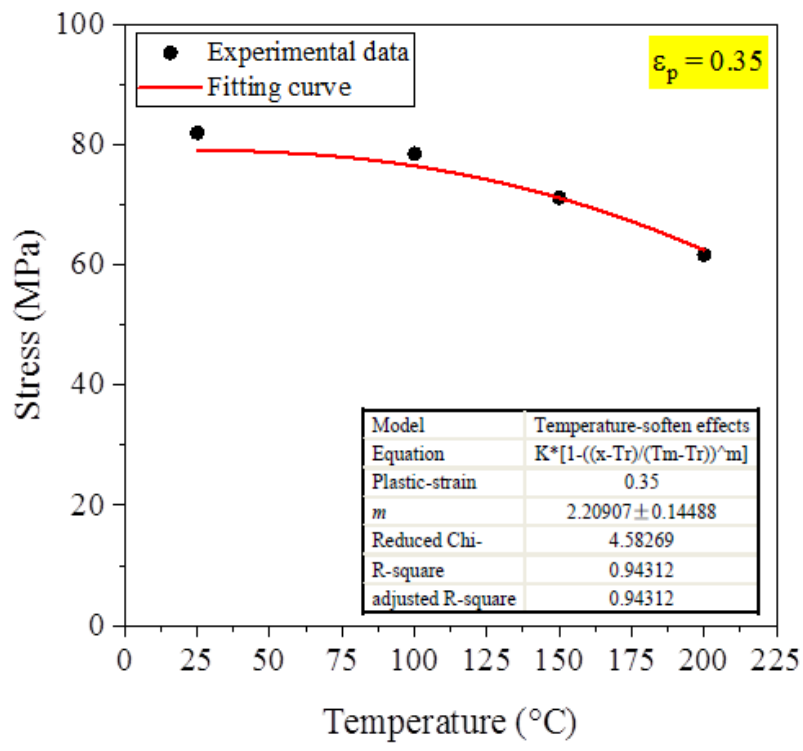

(b)

Figure 8. The fitting curves for the temperature-soften term at the fix plastic strain. (a) $\varepsilon_{p}=0.25$; (b) $\varepsilon_{p}=0.35$. 


\section{Conclusions}

Based on the new formulation proposed in the literature [11], the mechanical behaviors of the sintered reactive material $\mathrm{PTFE} / \mathrm{Al} / \mathrm{Si}$ are studied systematically. Fitting the JC model to the static and dynamic experimental data, all the parameters of which can be determined, the determined final form is

$$
\sigma_{y}=\left(13.0+5.949 \bar{\varepsilon}_{p}^{0.289}\right)\left(\dot{\varepsilon} / \dot{\varepsilon}_{0}\right)^{0.07963}\left[1-\left(\frac{T-T_{r}}{T_{m}-T_{r}}\right)^{2.20907}\right]
$$

which can be used to describe the static or dynamic mechanical behavior and be used as one basis for the future numerical simulation research.

\section{Acknowledgements}

This work is financially supported by the National Natural Science Foundation of China (grant nos. 11002162 and 11072262). The financial contributions are gratefully acknowledged.

\section{Conflicts of Interest}

The authors declare no conflicts of interest regarding the publication of this paper.

\section{References}

[1] Mock Jr., W. and Drotar, J.T. (2007) Effect of Aluminum Particle Size on the Impact Initiation of Pressed Ptfe/al Composite Rods. AIP Conference Proceedings, 955, 971-974. https://doi.org/10.1063/1.2833292

[2] Zhang, Y. and Li, H. (2008) Fluorine-Containing Functional Materials. Chemical Industry Press.

[3] Yang, S., Xu, S. and Zhang, T. (2008) Preparation and Performance of PTEF/Al Reactive Materials. Journal of National University of Defense Technology, 30, $39-42,62$.

[4] Ames, R. (2005) Energy Release Characteristics of Impact-Initiated Energetic Materials. MRS Online Proceedings Library, 896, 308. https://doi.org/10.1557/PROC-0896-H03-08

[5] Ames, R. (2005) Vented Chamber Calorimetry for Impact-Initiated Energetic Materials. Proceedings of 43 rd AIAA Aerospace Sciences Meeting and Exhibit. https://doi.org/10.2514/6.2005-279

[6] Ames, R.G. and Waggener, S.S. (2005) Reaction Efficiencies for Impact-Initiated Energetic Materials. Proceedings of Energetic Materials. Performance and Safety 36th and 32nd, Karlsruhe (DE). https://doi.org/10.2514/6.2005-279

[7] Cai, J., Jiang, F., Vecchio, K.S., Meyers, M.A. and Nesterenko, V.F. (2007) Mechanical and Microstructural Properties of PTFE/Al/W System. Proceedings of AIP Conf. Proc., 955, 723-726. https://doi.org/10.1063/1.2833220

[8] Cai, J., Walley, S.M., Hunt, R.J.A., Proud, W.G., Nesterenko, V.F., et al. (2008) High-Strain, High-Strain-Rate Flow and Failure in PTFE/Al/W Granular Composites. Mater. Sci. Eng. A-Struct. Mater. Prop. Microstruct. Process, 472, 308-315. https://doi.org/10.1016/j.msea.2007.03.068

[9] Osborne, D.T. (2006) The Effects of Fuel Particle Size on the Reaction of Al/Teflon 
Mixtures. Ph.D. Thesis, Texas Tech University.

[10] Mock Jr., W. and Holt, W.H. (2006) Impact Initiation of Rods of Pressed Polytetrafluoroethylene (PTFE) and Aluminum Powders. AIP Conference Proceedings, 845, 1097-1100. https://doi.org/10.1063/1.2263514

[11] Ran, X.W., Ding, L.L., Zhou, J.Y. and Tang, W.H. (2019) Research on the Energy Release Characteristics of Six Kinds of Reactive Materials. Materials, 12, 3940. https://doi.org/10.3390/ma12233940

[12] Johnson, G.R., Cook, W.H. and Johnson, G. (1983) A Constitutive Model and Data for Metals Subjected to Large Strains, High Strain Rates and High Temperatures. 7th International Symposium on Ballistics, 541-547.

[13] Xu, S.L. (2010) Study on the Mechanical Performance of Polytetrfluorethylene/Al Energetic Reactive Materials. PhD Thesis, National University of Defense Technology, Changsha. 